\title{
Arthroscopic Curettage of Intraosseous Ganglions of the Lunate Bone
}

\author{
Reza Shahryar Kamrani ${ }^{1}$; Leila Oryadi Zanjani ${ }^{1, *}$; Mohammad Hosein Nabian ${ }^{1}$ \\ ${ }^{1}$ Department of Orthopedic and Trauma Surgery, Shariati Hospital, Tehran University of Medical Sciences, Tehran, IR Iran \\ ${ }^{*}$ Corresponding author: Leila Oryadi Zanjani, Department of Orthopedic and Trauma Surgery, Shariati Hospital, Tehran University of Medical Sciences, Tehran, IR Iran. Tel: +98- \\ 9121017913, Fax:+98-2133542020, E-mail: leila_zanjani@yahoo.com
}

Received: March 10, 2014; Revised: May 20, 2014; Accepted: July 15, 2014

\begin{abstract}
Background: Intraosseous ganglions (IOGs) of the lunate bone are a rare cause of chronic wrist pain. Traditional treatment by open curettage and bone grafting can lead to ongoing pain and stiffness of the wrist.

Objectives: In this study, an arthroscopically assisted minimally invasive technique of debridement without grafting of the lunate IOG was presented.

Patients and Methods: In a prospective study, eight patients with symptomatic lunate intraosseous ganglions were treated with an arthroscopically assisted curettage technique without bone grafting in seven of them. At the preoperative examination and the last followup, wrist flexion/extension range of motion, the Mayo Wrist Performance Score, the Quick-Disabilities of the Arm, Shoulder, and Hand (Quick-DASH) score and the visual analog scale (VAS) were calculated. At final follow-up recurrence, patients' general satisfaction, return to work and complications were assessed.

Results: The mean age of patients was $37 \pm 8$ years. The mean duration of symptoms and follow-up were $12 \pm 4$ and $28 \pm 17$ months, respectively. The mean pretreatment wrist flexion-extension arc of motion was $151 \pm 46$, and $174 \pm 9$ at the last follow-up, which was not significantly different $(\mathrm{P}=0.23)$. All patients had statistically significant improvements in the Mayo functional wrist score $(\mathrm{P}<0.01)$, QuickDASH score $(\mathrm{P}<0.01)$ and VAS score $(\mathrm{P}<0.01)$.

Conclusions: Arthroscopic debridement of intraosseous ganglions of the Lunate bone without bone grafting could improve wrist functional outcomes with fewer complications.
\end{abstract}

Keywords:Wrist; Arthroscopy; Ganglion

\section{Background}

Diagnosis of intraosseous ganglion (IOG) cysts is based on specific radiographic findings and histological examination (1). Intraosseous ganglions have been reported most frequently in the lower extremity especially around the hip, knee, and ankle (2). Intraosseous ganglion cyst is an infrequent cause of chronic wrist pain. It is most commonly reported in the lunate and scaphoid bones (3-6). Its differential diagnosis includes osteoid osteoma, giant cell tumor, enchondroma, osteoblastoma and the Kienbock's disease (7). The etiology remains unknown. In symptomatic cases treatment includes open curettage with autologous or homologous bone grafting (8) resulting in a high rate (up to $40 \%$ ) of persistent symptoms in previous reports $(9,10)$. Ashwood et al. reviewed eight cases of intraosseous ganglions of the lunate treated by arthroscopic assisted drilling and autologous bone grafting and reported promising results (11).

\section{Objectives}

In this prospective study, we evaluated medium-term effects of a minimally invasive arthroscopic curettage technique without bone grafting in cases with symptomatic carpal bones intraosseous ganglia.

\section{Patients and Methods}

\subsection{Patients}

In a prospective study between February 2010 and October 2013, we treated wrist intraosseous ganglions with an arthroscopically assisted technique in eight patients (4 men and 4 women). The radiographic inclusion criterion was an eccentrically located radiolucent lesion with a thin sclerotic margin contained within and not expanding the bone (8-10) (Figure $1-\mathrm{A}, \mathrm{B}$ ). The dominant limb was involved in six patients. All patients presented with chronic wrist pain in the perilunate area unresponsive to a minimum of six months conservative treatment including immobilization and non-steroidal anti-inflammation medications and not finding other sources for the pain. The medical ethics committee of the orthopedics department of our hospital approved the study. An informed consent was obtained from all patients after explaining available treatment options prior to surgery.

\subsection{Assessments}

The patient was followed up at 3 and 12 weeks and 6 months. At preoperative examination and the last followup, following assessments were conducted. The wrist

Copyright (C) 2014, Iran University of Medical Sciences. This is an open-access article distributed under the terms of the Creative Commons Attribution-NonCommercial 4.0 International License (http://creativecommons.org/licenses/by-nc/4.0/) which permits copy and redistribute the material just in noncommercial usages, provided the original work is properly cited. 

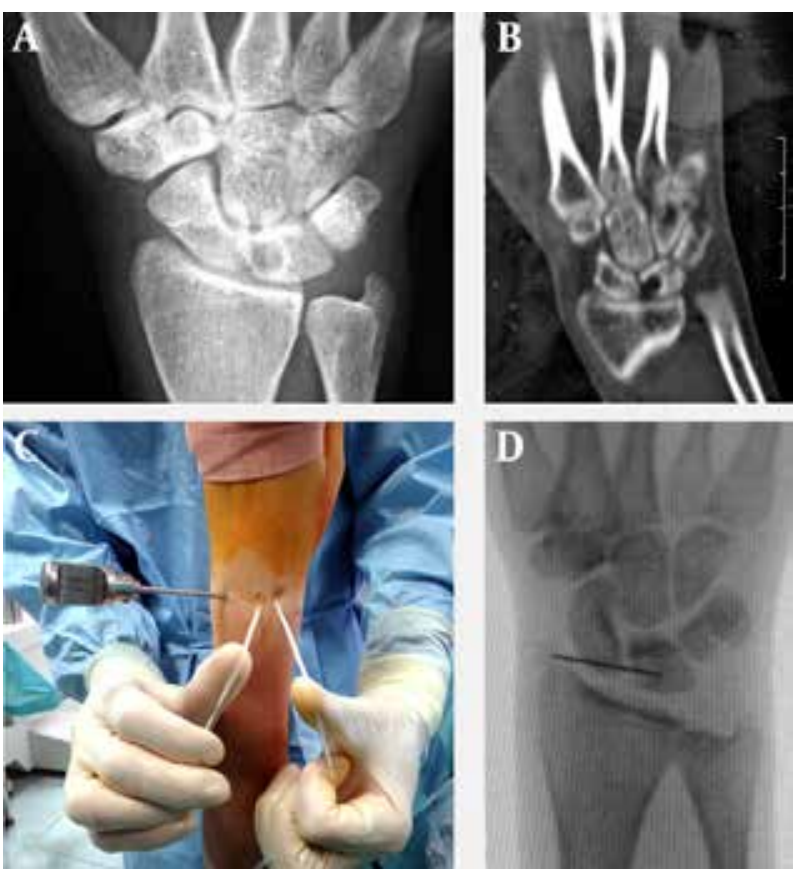

Figure 1. A) Radiographic Inclusion Criterion Was an Eccentrically Located Radiolucent Lesion With a Thin Sclerotic Margin Contained Within and not Expanding the Bone; B) Intraosseous Ganglion Site Was Identified Preoperatively by CT Scan Imaging; C) A Trans-Four Portal Was Created Just Direct to Lunate and Tendons Were Protected With Two Pair Tapes; D) Arthroscopic Burr Site Was Confirmed Intraoperatively by Fluoroscopic Images.

flexion/extension range of motion was measured by a goniometer. All patients were assessed with the Mayo Wrist Performance Score, the Quick-Disabilities of the Arm, Shoulder, and Hand (Quick-DASH) score and the visual analog scale (VAS). At the final follow-up recurrence, patients' general satisfaction, return to work and complications were assessed. Patients were assessed postoperatively by radiography to assess intralesional trabeculation at the same time of their clinical evaluation.

\subsection{Operative Technique}

The procedure was performed in supine position under general anesthesia using a tourniquet. The arm was suspended with Chinese finger traps and the elbow flexed to $90^{\circ}$ with the wrist in a vertical traction $4.5 \mathrm{~kg}$. We used a 2.4-mm arthroscope with a visual angle of $30^{\circ}$ and a wet technique with normal saline solution provided through the arthroscope cannula. Initial diagnostic wrist arthroscopy was performed via standard 3/4 and 6R portals and intra-articular findings were documented. Lunate and intraosseous ganglion sites were identified preoperatively by radiography and CT scan imaging, which were later confirmed intraoperative by arthroscopic land marks (scapholunate ligament) and fluoroscopic images. For lunate intraosseous ganglions, a trans-four portal was created just direct to lunate. The tendons were protected with two pair tapes (Figure $1-\mathrm{C}$ ). The exact site of the cyst was marked by a pin and confirmed under C-arm fluoroscopy (A A 3-mm sheath-less burr was introduced trough trans-four portal toward the cyst and it was destroyed by rotating motion of the burr without bone grafting. Finally, arthroscopy was used to inspect the hole, the joint was irrigated and the wounds were sutured with absorbable sutures. One patient (case No.7) asked us for filling the defect with autogenous bone graft. In this patient, we harvested cancellous bone graft from iliac crest and inserted it to the excised ganglion trough trans-four cannula.

\subsection{Postoperative Rehabilitation Program}

No splinting was used postoperatively. Wrist range of motion began on the first postoperative day. The patient was followed up at 3 and 12 weeks and 6 months.

\subsection{Statistical Analysis}

Statistical analysis was performed by SPSS software, version 18.0 (Statistical Product and Service Solutions, SPSS Inc., Chicago, IL, USA). Data was presented as the mean \pm standard deviation. Paired T-test was used to compare quantitative variables before and after the operation. $\mathrm{P}<$ 0.05 was considered statistically significant.

\section{Results}

We treated eight cases; four patients were housekeepers, two were laborers and two were surgeons. Seven patients participated in follow-up. The mean age of observed patients was $37 \pm 8$ years (ranged $29-48$ years). The mean duration of symptoms was $12 \pm 4$ months (ranged 7-18 months). The mean duration of follow-up was $28 \pm 17$ months (ranged 9-51 months). The mean wrist flexionextension arc of motion pretreatment was $151 \pm 46$, and the last follow-up was $174 \pm 9$, which was not significantly different $(\mathrm{P}=0.23)$ (Table 1$)$.

According to the Mayo functional wrist scores, there were four excellent, two good and one satisfactory result at the final follow-up. All patients had statistically significant improvements in the Mayo functional wrist score (P $<0.01)$, the Quick-DASH score $(\mathrm{P}<0.01)$ and VAS score $(\mathrm{P}<$ $0.01)$. Details are presented in Table 2.

There were no minor or major complications regarding arthroscopic procedure except transient superficial ulnar nerve paresthesia in one patients. None of patients experienced recurrence or underwent another operation. All patients were satisfied with the operation result. Two patients including one who underwent bone grafting complained about mild discomfort and residual pain differing in nature with the previous pain. All patients returned to their previous occupation and achieved normal function. At final follow-up, radiographic analysis showed no sign of collapse, fracture or recurrence in lunate area. However, trabeculation within the intraosseous lesion was evident in one patient who had bone graft and partial trabeculation in two other ones (Figure 2). 
Kamrani RS et al.

\begin{tabular}{|c|c|c|c|c|c|c|c|c|}
\hline Patients' Data & Case 1 & Case 2 & Case 3 & Case 4 & Case 5 & Case 6 & Case 7 & Case 8 \\
\hline Age, y & 30 & 36 & 29 & 38 & 37 & 48 & 32 & 48 \\
\hline Gender & Male & Female & Male & Female & Male & Female & Male & Female \\
\hline Duration of symptoms, mo & 9 & 12 & 12 & 12 & 12 & 18 & 7 & 18 \\
\hline Occupation & Laborer & At home & Laborer & At home & At home & Surgeon & Surgeon & At home \\
\hline Bone graft & No & No & No & NO & No & No & Yes & No \\
\hline Duration of follow-up, mo & 21 & 50 & 9 & 25 & b & 29 & 11 & 51 \\
\hline Preop/final VAS score & $7 / 1$ & $10 / 2$ & $8 / 1$ & $9 / 1$ & $\left.9\right|^{\mathrm{b}}$ & $10 / 1$ & $9 / 3$ & $10 / 1$ \\
\hline $\begin{array}{l}\text { Preop/final wrist range of motion, } \\
\text { degree }\end{array}$ & $160 / 160$ & $120 / 160$ & $180 / 180$ & $180 / 180$ & $150 /-b$ & $180 / 180$ & $60 / 180$ & $180 / 180$ \\
\hline Preop/final quick-DASH score & $47.7 / 4.5$ & $65.9 / 22.7$ & $43.2 / 0$ & $75 / 6.8$ & $\left.71\right|_{-} ^{\mathrm{b}}$ & $90.9 / 4.5$ & $86.4 / 13.6$ & $86.4 / 6.8$ \\
\hline Preop/final Mayo wrist score, points & $75 / 100$ & $25 / 85$ & $50 / 100$ & $50 / 90$ & $40 /-^{\mathrm{b}}$ & $50 / 100$ & $15 / 85$ & $25 / 90$ \\
\hline $\begin{array}{l}\text { Trabeculation status in the last radiog- } \\
\text { raphy }\end{array}$ & Partial & None & None & None & b & Partial & Complete & None \\
\hline General satisfaction with the operation & Yes & Yes & Yes & Yes & $\mathrm{b}$ & Yes & Yes & Yes \\
\hline Complication & Ulnar Paresthesia & None & None & None & b & None & None & None \\
\hline
\end{tabular}

a Abbreviations: DASH, disabilities of the arm, shoulder, and hand; Preop, preoperative; VAS, visual analog scale.

$\mathrm{b}$ Missing data due to lack of patients' cooperation.

Table 2. Comparing Preoperative and Final Assessments ${ }^{a, b}$

\begin{tabular}{lccc}
\hline Scores/Measurements & Preoperative & Final Follow-Up & Improvement \\
\hline Wrist flexion-extension arc of motion, degrees & $151 \pm 46$ & $174 \pm 9$ & $22 \pm 45$ \\
Mayo functional wrist score & $41 \pm 20$ & $93 \pm 7$ & $51 \pm 15$ \\
Quick-f score & $70.8 \pm 19$ & $8.4 \pm 7.5$ & $62 \pm 19$ \\
VAS score & $9 \pm 1.1$ & $1.1 \pm 0.4$ & $7.8 \pm 1$ \\
\hline
\end{tabular}

a Abbreviations: DASH, disabilities of the arm, shoulder, and hand; VAS, visual analog scale.

$\mathrm{b}$ Data are presented as Mean \pm SD.

\section{Figure 2. Last Follow-up Radiography}
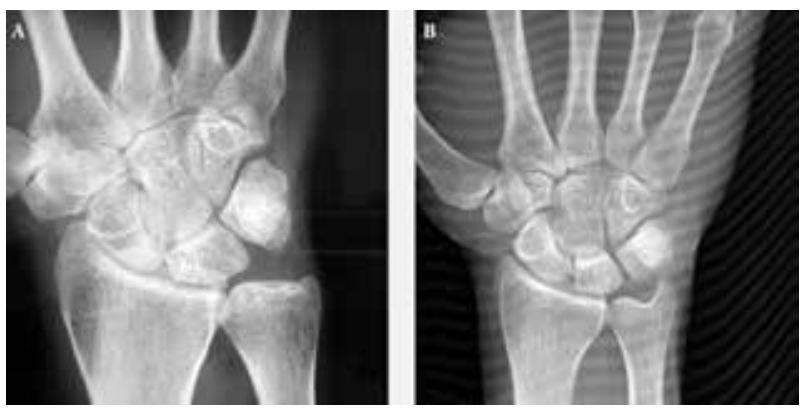

A, Complete Trabeculation Within Intraosseous Ganglion (Case No.7); B, Partial Trabeculation (Case No.6).

\section{Discussion}

"Intraosseous Ganglion” (IOG) term was first used in 1966 by Crabbe to describe synonymous terms including synovial bone cyst, juxta-articular bone cyst, ganglionic cystic defect of bone and subchondral bone cyst (12). It can be distinguished from other common differential diagnoses of carpal bone lytic lesions using imaging modalities including radiography, CT scan and bone scan (7). IOG cyst might be completely asymptomatic or clinically manifested by chronic pain unresponsive to analgesic medications. This pain could result from intraosseous hyper pressure secondary to pathologic process development within an inextensible limited cavity which based the treatment philosophy (13). Surgery is indicated in case of symptoms affecting function resistant to conservative treatment after at least six months with no other recognized sources of pain or in the presence of radiographic progressive signs $(9,14)$. Currently, the treatment preference is intralesional curettage of the IOG cyst associated with autologous bone grafting to prevent any recurrence and the risk of bone collapsing and fracture $(9,10,13)$. However, previous studies with open curettage and bone grafting procedures have involved heterogeneous and unpredictable outcomes and high complication rates $(2,9,10,15)$. In 2003, Ashwood suggested a new technique in arthroscopically assisted treatment of IOG of the Lunate in eight cases. They reported six good or excellent wrist functional results and two fair results 
with fewer complications using drilling and autologous bone grafting. They proposed that improved results with arthroscopic technique could be due to less arthrofibrosis because of minor damages to ligamentous structures using a small incision (11). Curettage of the cyst and packing with cancellous bone graft were performed in most reported cases to minimize the risk of recurrence, bone collapsing and fracture $(9,10)$. However, the technique of bone grafting involves operative harvest from a donor site having several complications, including painful scar, infection, hematoma, fracture, and gait disturbances (16). Therefore, several investigators proposed treatment of benign bone tumors by simple curettage without bone grafts and reported promising results (17-20). In the current study, using a minimally invasive arthroscopic excision technique without bone grafting, most patients had eventual relief of symptoms and pain with four excellent, two good and one satisfactory wrist Mayo function result. There were no recurrences, complications or reoperation at a mean of 2-year follow-up. At the final follow-up, one patient (case No. 7) showed trabeculation at the site of previous IOG and partial trabeculation was evident in two other patients, but there was no evidence of recurrence or bone collapse in radiographic analysis of all patients. Two of our patients complained residual pain around the portals. This study had some limitations. The follow-up time was short. They were limited cases. It was not practical to evaluate our patients histologically to confirm the diagnosis due to insufficient sampling during arthroscopic method. We could not compare the effect of bone graft, but we found that bone graft is not necessary for satisfactory results at least in medium follow up. According to the results of our study, arthroscopic debridement of intraosseous ganglions of the carpal bones without bone grafting could improve functional outcomes with less potential complications.

\section{Authors' Contributions}

Study concept and design: Reza Shahryar Kamrani; Analysis and interpretation of data: Reza Shahryar Kamrani, Leila Oryadi Zanjani and Mohammad Hosein Nabian; Drafting of the manuscript: Leila Oryadi Zanjani and Mohammad Hosein Nabian; Critical revision of the manuscript for important intellectual content: Reza Shahryar Kamrani, Leila Oryadi Zanjani and Mohammad
Hosein Nabian; Statistical analysis: Leila Oryadi Zanjani and Mohammad Hosein Nabian; Study supervision: Reza Shahryar Kamrani.

\section{References}

1. Chantelot C, Laffargue P, Masmejean E, Peltier B, Barouk P, Fontaine C. [Fracture of the scaphoid carpal bone secondary to an intraosseous cyst. Apropos of a case]. Chir Main.1998;17(3):255-8.

2. Schajowicz F, Clavel Sainz M, Slullitel JA. Juxta-articular bone cysts (intra-osseous ganglia): a clinicopathological study of eighty-eight cases. J Bone Joint Surg Br. 1979;61(1):107-16.

3. Bauer TW, Dorfman HD. Intraosseous ganglion: a clinicopathologic study of 11 cases. Am J Surg Pathol.1982;6(3):207-13.

4. Eiken O, Jonsson K. Carpal bone cysts: a clinical and radiographic study. Scand J Plast Reconstr Surg. 1980;14(3):285-90.

5. Iwahara T, Hirayama T, Takemitu Y. Intraosseous ganglion of the lunate. Hand.1983;15(3):297-9.

6. Uriburu IJ, Levy VD. Intraosseous ganglia of the scaphoid and lunate bones: report of 15 cases in 13 patients. J Hand Surg Am. 1999;24(3):508-15.

7. Nazerani S, Ebrahimpour A, Najafi A, Shams Koushki E. Intraosseous ganglion cyst of the lunate. Trauma Mon. 2012;16(4):198-200.

8. Helwig U, Lang S, Baczynski M, Windhager R. The intraosseous ganglion. A clinical-pathological report on 42 cases. Arch Orthop Trauma Surg. 1994;114(1):14-7.

9. Tham S, Ireland DC. Intraosseous ganglion cyst of the lunate: diagnosis and management. J Hand Surg Br.1992;17(4):429-32.

10. Waizenegger M. Intraosseous ganglia of carpal bones. $J$ Hand Surg Br. 1993;18(3):350-5.

11. Ashwood N, Bain GI. Arthroscopically assisted treatment of in traosseous ganglions of the lunate: a new technique.J Hand Surg Am. 2003;28(1):62-8.

12. Crabbe WA. Intra-osseous ganglia of bone. BrJSurg. 1966;53(1):157.

13. Mnif H, Koubaa M, Zrig M, Jawahdou R, Sahnoun N, Abid A. Ganglion cyst of the carpal navicular. A case report and review of the literature. Orthop Traumatol Surg Res. 2010;96(2):190-3.

14. Calcagnotto G, Sokolow C, Saffar P. [Intraosseus synovial cysts of the lunate bone: diagnostic problems]. Chir Main. 2004;23(1):1723.

15. Kambolis C, Bullough PG, Jaffe HI. Ganglionic cystic defects of bone.J Bone Joint Surg Am. 1973;55(3):496-505.

16. Gershuni DH, Pinsker R. Bone grafting for nonunion of fractures of the tibia: a critical review. J Trauma.1982;22(1):43-9.

17. Kamrani RS, Shafiei H, Shafieian L. Curettage Alone with no Graft in Enchondroma of Hand. Iran J Orthop Surg. 2012;38:7-12.

18. Hirn M, de Silva U, Sidharthan S, Grimer RJ, Abudu A, Tillman RM et al. Bone defects following curettage do not necessarily need augmentation. Acta Orthop. 2009;80(1):4-8.

19. Yanagawa T, Watanabe H, Shinozaki T, Takagishi K. Curettage of benign bone tumors without grafts gives sufficient bone strength. Acta Orthop. 2009;80(1):9-13.

20. Goto T, Yokokura S, Kawano H, Yamamoto A, Matsuda K, Nakamura K. Simple curettage without bone grafting for enchondromata of the hand: with special reference to replacement of the cortical window. J Hand Surg Br. 2002;27(5):446-51. 